\title{
Integrated multimedia electronic patient record and graph-based image information for cerebral tumors
}

\author{
John Puentes ${ }^{\mathrm{a}, \mathrm{b}, *}$, Bénédicte Batrancourt ${ }^{\mathrm{c}}$, Jamal Atif ${ }^{\mathrm{c}}$, Elsa Angelini ${ }^{\mathrm{c}}$, Laurent Lecornu ${ }^{\mathrm{a}, \mathrm{b}}$, \\ Abdelhamid Zemirline $^{\mathrm{a}, \mathrm{b}}$, Isabelle Bloch ${ }^{\mathrm{c}}$, Gouenou Coatrieux ${ }^{\mathrm{a}, \mathrm{b}}$, Christian Roux ${ }^{\mathrm{a}, \mathrm{b}}$ \\ a Institut TELECOM; TELECOM Bretagne, GET, Département Image et Traitement de l'Information, Brest, France \\ ${ }^{\mathrm{b}}$ INSERM, U650, Laboratoire de Traitement de l'Information Médicale, Brest, France \\ ${ }^{\mathrm{c}}$ Institut TELECOM; TELECOM Paris Tech, Département Traitement du Signal et des Images, CNRS UMR 5141 LTCI Paris, France
}

Received 24 January 2007; accepted 14 January 2008

\begin{abstract}
Current electronic patient record (EPR) implementations do not incorporate medical images, nor structural information extracted from them, despite images increasing role for diagnosis. This paper presents an integration framework into EPRs of anatomical and pathological knowledge extracted from segmented magnetic resonance imaging (MRI), applying a graph of representation for anatomical and functional information for individual patients. Focusing on cerebral tumors examination and patient follow-up, multimedia EPRs were created and evaluated through a 3D navigation application, developed with open-source libraries and standards. Results suggest that the enhanced clinical information scheme could lead to original changes in the way medical experts utilize image-based information.
\end{abstract}

Keywords: Multimedia electronic patient record; Anatomical graph; Neurology; Open-source software; Computerized knowledge-supported medical practice

\section{Introduction}

Designing electronic patient record (EPR) systems raises new challenges for integrating complex, evolving information contents and supports, as well as deciding what data format should be handled by healthcare information systems. Initially conceived to support healthcare costs analysis, the EPR has been expanding to integrate other kinds of data, in order to cope with hospital administration and medical practitioners' activity. Recently, emerging patient centred systems [1] that attempt to consolidate all relevant medical information, illustrate how both the quantity and the types of patient data needed for this EPR type are significantly increased compared to usual models. Moreover, computerized knowledge-supported medical practice and medical image processing interpretation tools generate new pieces of information, not taken into account

\footnotetext{
* Corresponding author at: Institut TELECOM; TELECOM Bretagne, GET, Département Image et Traitement de l'Information, Brest, France.

Tel.: +33229001339.

E-mail address: John.Puentes@telecom-bretagne.eu (J. Puentes)
}

by existing EPR applications. Initiatives to partially handle the changing content and data formats to be included into an EPR have been carried out in the form of isolated, disparate, and dedicated proprietary systems like hospital information system (HIS), clinical information system (CIS), picture archiving and communication systems (PACS), radiology information system (RIS), or EPR, among others, which deal autonomously with interdependent data subsets, being unable to interoperate. Even though standardization (i.e. health level seven - www.hl7.org) and industrial initiatives (i.e. integrating the healthcare enterprise-www.ihe.net) propose some solutions to the interoperability problem between proprietary applications, current systems remain locked with restricted or inexistent autonomous expansion possibilities.

Regardless of the actual incremental use of EPRs in HISs, and various efforts to conceptualize models adapted to clinical practice [2-5], a considerable amount of effort is still required to design new EPR-based systems that provide utilities beyond data listing tools [6]. An illustration of the research and development efforts required in designing new EPR systems is the need to integrate complex multimedia medical data, such 
as text, image data volumes and sequences, pathology-specific knowledge, as well as 2D and 3D anatomical representations, in a single EPR application. For instance, even if images generated in clinical practice are stored by PACS along with 2D or 3D anatomical representations such as segmentation processing results, parts of the associated patient data are separately stored in the RIS and the respective specialty EPRs $[7,8]$. More sophisticated approaches intend: to provide remote visualization access to PACS images along with RIS patient data [9], or combined PACS-HIS-CIS data [10], using a client web interface, to integrate data management, transactions and user interfaces in a scalable emergency department information system [11], or to integrate some selected pieces of information (work list, reports and image visualization) from HIS and PACS [12]. Among the various multimedia medical data types, images and their associated computer-based interpretation reports are strongly involved in a majority of diagnosis procedures, because of their capability to allow for visualization and quantification of the anatomy and pathological findings, as well as the activity of multiple pathological processes. Furthermore, image-based interpretations and annotations are essential for therapeutic planning and patient follow-up. Despite their recognized importance, medical images, along with their segmentation results and the associated anatomical and functional knowledge, have not been yet fully integrated into conventional EPR structures.

This paper presents a unified approach that addresses the multimedia EPR domain of this large problematic, taking advantage of a detailed user requirements comprehension, and focusing on the integration into the EPR of the following components:

1. Prior knowledge specific to the anatomy, the function and the pathologies under study.
2. Medical imaging data.

3. Information extracted from medical images, after image segmentation.

In the context of this paper, neuroimaging provides comprehensive neurological diagnosis information for the characterization of morphological and biological alterations of cerebral structures, tumor grades and growth patterns, as well as tumor response to treatments and patient prognosis [13].

A methodological framework is proposed to construct a specialized neurological EPR, including patient-specific imaging data and graph-based information, derived from a generic anatomical model and knowledge base. Fig. 1 illustrates the EPR system functional scheme. An adapted graphic user interface (GUI) queries and visualizes the different EPR elements: 2D or 3D images, 2D or 3D segmented images, graph information, and patient data, depending on user interactions. The proposed implementation makes use of open software libraries, standards, and clinical ontologies, which are emerging paradigms gaining wide international acceptance [14]. Beyond the challenge of building a more comprehensive EPR data structure and content, it is also important to consider the potential EPR enhanced capability for reviewing clinical information extracted from medical images, toward an integrated therapeutic patient follow-up tool. Those enhanced capabilities concern only diagnosis support tasks, excluding functionalities like workflow or patient management.

A neurological examination scenario, related to brain tumors diagnosis based on the interpretation of magnetic resonance imaging (MRI) datasets, acquired with multiple protocols such as T1-weighted and T2-weighted, has been chosen to design the EPR framework and evaluate the 3D navigation application prototype. Based on a generic architecture, the

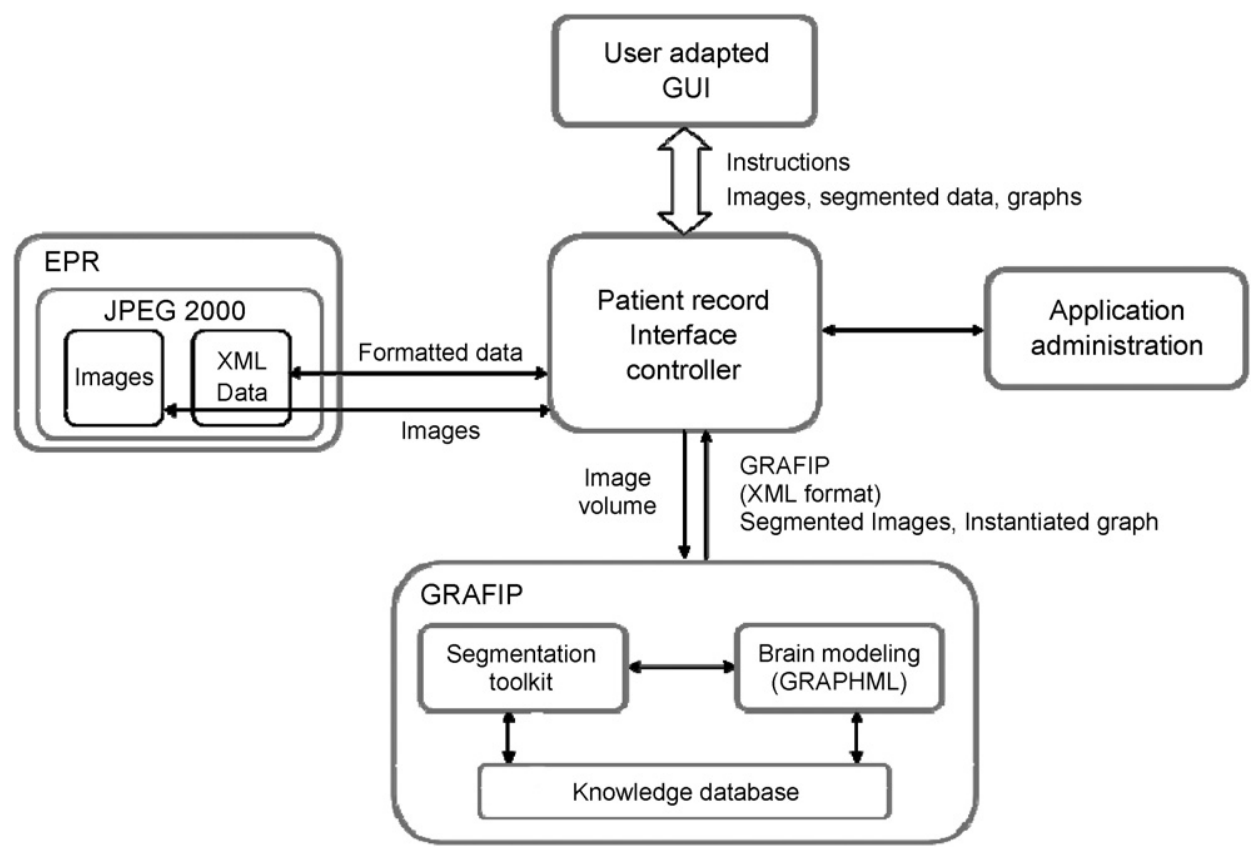

Fig. 1. Functional scheme of the proposed EPR application. 
integration of image-based information in the EPR is performed by a flexible system, capable of supporting the implementation of "pathology-dependent" modules, enabling, for example the identification, grouping, and processing of specific cases according to pathology evolving parameters, or allowing the specialist to examine groups of similar cases. Another aspect is that the segmentation methods we use are pathology-dependent (see Section 3). The proposed EPR system prototype is intended to:

1. Provide a rich and flexible GUI for enhanced interaction with the relevant and structured EPR multimedia content.

2. Display $2 \mathrm{D}$ or $3 \mathrm{D}$ brain structures segmentation results, combined with the respective tumor and MRI volume.

3. Associate segmentation results to anatomical knowledge, through spatial localizations of the tumor and surrounding segmented cerebral structures, as well as the positioning of the tumor on functional cerebral maps.

The main contribution of this paper is the development of a multimedia neurological EPR capable of integrating patient data, MRI segmentation results, and graph-based representations of the patient's cerebral anatomy, along with the implementation of a flexible visualization and navigation tool for combined image and graph data. The EPR multimedia structure also offers the possibility to compare information extracted from a large repository of EPRs with correlated pathologies, for example through non-hypothesis driven insights generation [15]. The different elements of the multimedia EPR are described in Section 2, while brain anatomy modeling and image segmentation methods are presented in Section 3. The EPRbased system design is detailed in Section 4. Discussion and conclusions are outlined in Section 5.

\section{Multimedia EPR}

Medical data are inherently multimedia, encompassing digital recording of signals, images, image sequences and image volumes, examination and laboratory results, and diagnosis and therapy follow-up reports. Computer-based processing of this data generates additional media objects that provide information and knowledge to support medical practice. Data processing tools include events detection on signals, image segmentation, anatomical modeling and reconstruction, or databases annotation. For data integration, clinical applications that exploit EPR multimedia data conventionally rely on a network infrastructure, data storage devices, visualizationprocessing workstations, and tailored applications, eventually connected to medical data acquisition equipments. Besides, depending on the workload and the targeted usage, a medical multimedia EPR setup can execute intra or inter-hospital data generation, storage, and processing, for signal, image, or combined applications.

An increasing amount of systems are based on client-server architectures, given the predominant requirement of distributed data exploitation. Until now, even if a significant variety of multimedia and network technologies are available to acquire, transfer and analyze medical multimedia information, medical data acquisition equipments offer seldom the possibility to be simply integrated with a variety of emerging technologies. For example PACS, based on images centralized storage, and initially designed to reduce and eventually eliminate the need of film supports for medical imaging examinations [16], lack of multiple complementary generic functionalities essential in a full multimedia application, where interoperable distributed storage and use of compatible dynamic multimedia documents are required $[9,12]$. At a higher level, following data acquisition, physical storage and transfer, efficient approaches for sophisticated data exploitation remain a complex issue. Our work addresses part of this problem on the client side, assuming that image data can be read at an intermediation storage server.

\subsection{Multimedia EPR content}

The proposed EPR core follows a generic model based on emerging multimedia standards, combined to cope with the design of a neurological multimedia EPR system for tumor diagnosis based on MRI examinations. It consists of personal, historical, and clinical patient data, associated to one or several MRI image volumes, on which a cerebral tumor and some of the neighboring healthy anatomical structures have been segmented. The extracted information is organized in a patientspecific graph representing the anatomical structures and their spatial relations. This section describes the main elements in some detail.

\subsubsection{Image volumes}

Medical image compression algorithms still bear strong limitations in terms of tradeoffs between image quality preservation and compression performance, which have prevented the inclusion of large medical imaging volume data in EPR files $[17,18]$. To address this issue, we have opted for the open standard JPEG-2000 [19] image compression scheme, rather unknown in the medical realm. Compared to the existing proprietary solutions and standards, JPEG-2000 targets the need for greater image compression with enhanced flexibility and efficient interchangeability, for different types of still images (bi-level, graylevel, color, and multi-component, from $32 \times 32$ to $10 \mathrm{~K} \times 10 \mathrm{~K}$ pixels, with as much as 16.384 components, and a precision depth of up to 38 bits/component), handled by different imaging exploitation modalities. Based on wavelet transforms, it offers interesting features like: (1) significantly improved low bit-rate performance useful for network image transmission; (2) compression and decompression of images with variable dynamic ranges for each color component; (3) lossless compression to preserve data integrity, with enhanced performance compared to other standards; (4) image reconstruction with different resolutions depending on the application; (5) compression of arbitrary shaped user defined regions of interest, having less distortion than the rest of the image; (6) robustness to bit errors in wireless communication channels; and (7) contentbased description in XML (eXtensible Markup Language) to facilitate data search or compressed images description. 


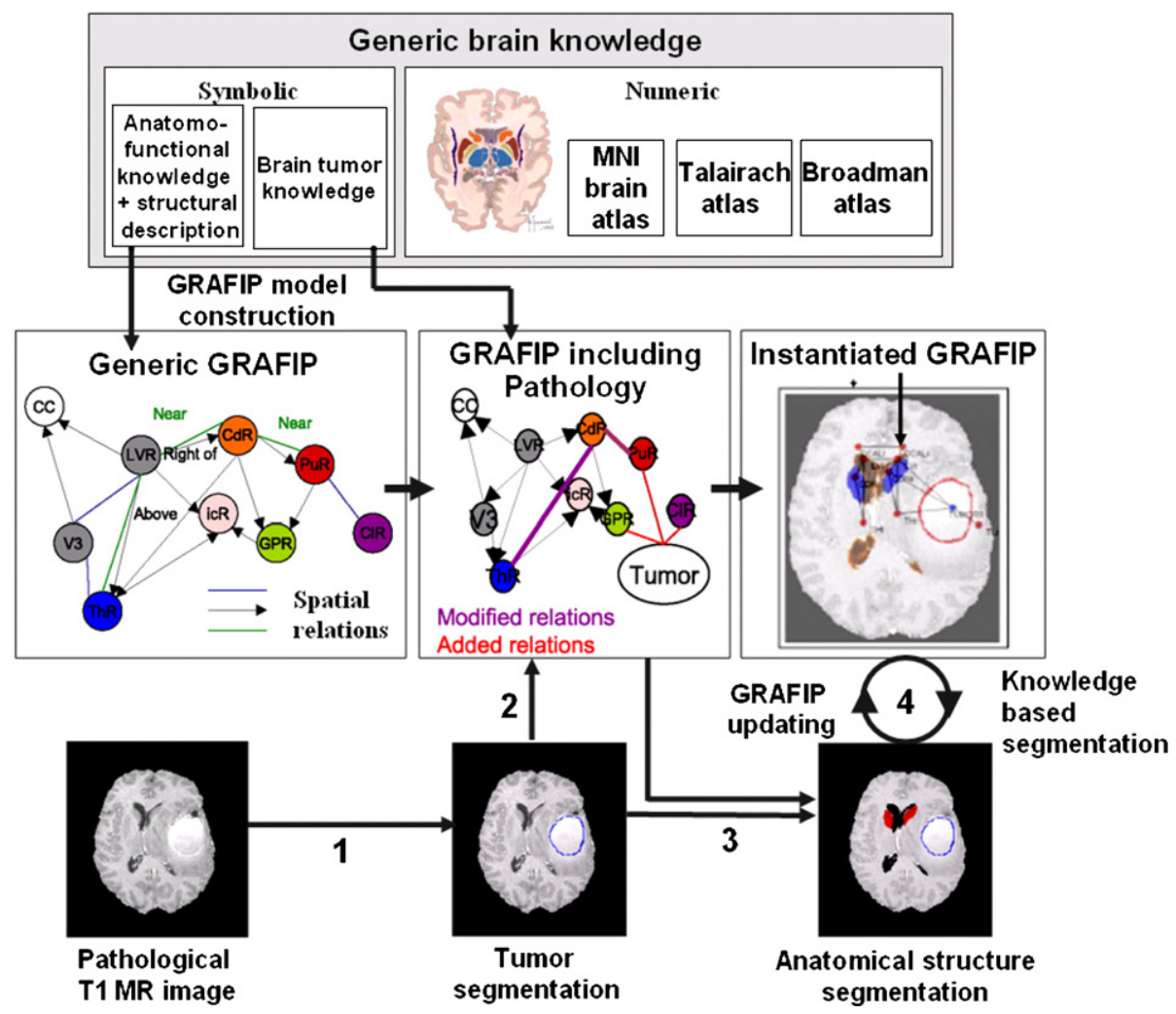

Fig. 2. Overview of the GRAFIP framework: modeling of the normal brain anatomy in a generic GRAFIP and iterative building of a patient-specific GRAFIP from segmented structures on MRI data.

Therefore, the basic EPR model has been defined as:

- One exam containing a JPEG-2000 lossless compressed image volume, originally in native DICOM format.

- The corresponding patient data formatted using XML, which can be either encapsulated in the same code stream [20], or stored separately. In the first case XML metadata is easily extracted without decoding the image, while in the second, images and patient data are linked in the database. Both solutions allow handling autonomous content objects, in a simple and modular manner. Besides, sub-tags structure and content defined by user requirements can be adapted in a flexible way to other medical specialties.

\subsubsection{Patient-specific image-based information}

A generic cerebral anatomical model combined with a knowledge database has been built and structured as a graph. This graph, once specified for each patient, becomes a graph of representation of anatomical and functional information for an individual patient including pathologies (GRAFIP). Section 3 describes how the GRAFIP framework provides the data structure applied to represent and integrate image-based information in the EPR system.

In the context of cerebral oncology, the process of generating a patient-specific GRAFIP, combining MRI image segmentation results with a generic cerebral GRAFIP, is illustrated in Fig. 2. Given the segmented structures, the generic GRAFIP is instantiated according to:

- The positioning of the anatomical structures, encoded in the graph nodes.

- The shape characteristics of these structures, encoded in the graph node attributes, and the graph edges via spatial relations.

\subsection{Data model}

Based on a series of discussions with three neurosurgeons regarding their data handling tools, and a set of standalone groups of independent tables from a collaborating hospital, a structured EPR data model was defined. It contains around 300 original data elements, structured in an integrated model, on which we added pointers to explicitly link images and their related diagnosis reports to the multimedia EPR. The obtained data model is formatted using XML, according to an XSD (XML Schema Description) model file. Fig. 3 provides an overview of the proposed data model that articulates the EPR structure.

All EPR items are grouped in four parts: general administrative and demographic data, medical examinations, treatments and follow-up, detailed below.

\subsubsection{General administrative and demographic data}

This part provides an easy to consult administrative and demographic patient data summary. Administrative items are 


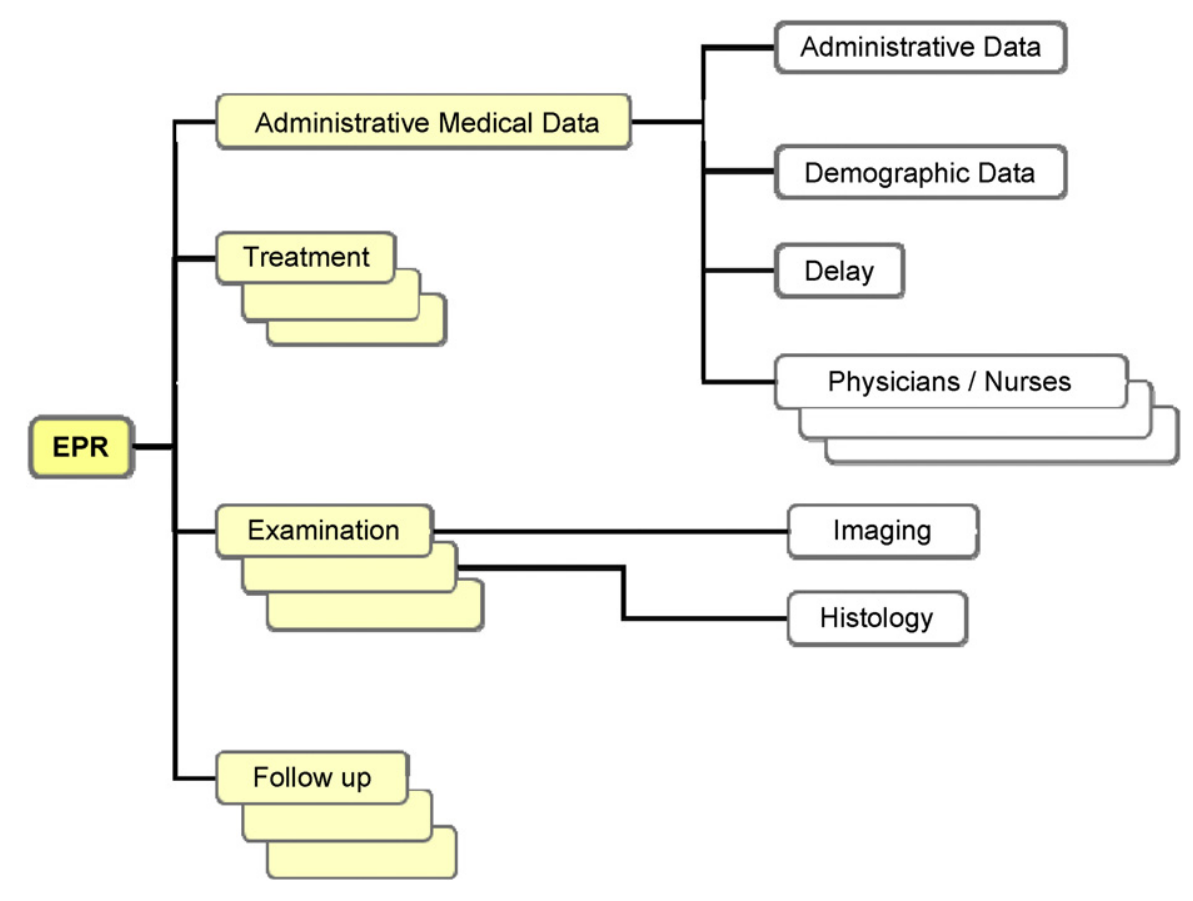

Fig. 3. Articulation of the different EPR data model components.

defined using fields including last and first names, patient identification code, address, telephone, birth date, insurance, etc. Demographic data give information about the pathology (type and class), weight, height, quality of life, allergies, particular remarks, antecedents (neurological, family clinical background information), etc. Additionally, there is information concerning the delay between different treatments, the healthcare protocol, as well as physicians and nurses' information (including names, addresses, professional activity, telephone numbers, etc.).

\subsubsection{Medical examinations}

This list contains records from all the examinations that the patient has undergone in the past. Two examination types are defined in the data structure: imaging and histological. A complete description of each one is combined with their respective clinical report. In the particular case of an imaging examination, an explicit field provides the link to the image volume data. The imaging examination is described by items such as date, imaging protocols (MRI, DTI, angiography, etc.), lesion size change, presence of edema or cyst, anatomical and functional localization of the pathology, its extension, the corresponding image volume identification, the related GRAFIP, etc. Histological examinations are described by items including date, histology examination number, frozen sample number, performed surgical procedures (biopsy, removal, and unknown), diagnosis, identified biological molecules, remarks, etc.

\subsubsection{Treatments}

This list describes the different treatments undergone by the patient. Four treatment types have been defined in the case of cerebral oncology: surgery, chemotherapy, X-ray radiation alone, or associated with chemotherapy. A set of items (includ- ing date, treatment type, referent physician, motive, etc.) are common to all treatments. In addition, some information concerns the treatment effects on the patient.

\subsubsection{Follow-up}

This list provides complementary information regarding the patient's pathology evolution after the treatment, taking into account the reports from several examinations. The main items concern: type of treatment, follow-up episodes dates, circumstances, observed clinical evolution, hospitalization reasons and dates, epilepsy crises and associated treatments, examinations carried out and their respective date, follow-up reports and additional remarks, clinical and paraclinical reports, etc.

\section{Brain anatomy modeling and image segmentation}

Generic knowledge and individual information extracted from medical imaging exams have been modeled applying the GRAFIP [21] framework. It enables to modify a generic anatomical knowledge model, adapting it to a specific clinical case, including pathological areas. This modification is performed via the integration of information extracted from the segmentation of medical imaging exams.

The GRAFIP framework is based on an attributed relational graph representation. Vertices of the graph encode the cerebral structures while the edges encode the matter type of vertices, as well as their spatial organization and spatial relations. More specifically, the GRAFIP is built with a hypergraph structure, where hyperedges describe relations between an arbitrary number of vertices. 


\subsection{Graph-based generic model}

This section describes the hypergraph structure of the GRAFIP.

- Vertices represent cerebral structures. According to the preidentified needs from a group of collaborating clinicians, 622 cerebral structures have been selected and extracted from the Neuronames Brain Hierarchy (NBH) which is a comprehensive hierarchical nomenclature for structures of the human brain [22].

- Hyperedges represent relations between vertices. Our model includes major types of anatomical relations according to the normal cerebral anatomy. A set of 651 relations was encoded to model anatomical links between various cerebral structures.

- Hierarchical relations: The anatomical model includes 596 taxonomical relations, also based on the NBH.

- Spatial relations complement the descriptive graph, with a set of 55 spatial relations. Hyperedges are particularly interesting when dealing with complex spatial relations involving more than two structures. For instance, the posterior limb of the internal capsule separates the putamen from the thalamus.

The hypergraph structure of the GRAFIP is illustrated in Fig. 2. Vertices and edges possess attributes [21] that deal with anatomical characteristics (structure location, shape, and volume), generic anatomo-functional knowledge according to the Brodmann's cytoarchitectonic referential [23] and tissue alterations (normal and pathological).

\subsection{GRAFIP encoding}

A GRAFIP is built in three stages:

1. Loading of a generic anatomo-functional model of the human brain.

2. Encoding of the generic model in a hypergraph structure.

3. Iterative instantiation of the hypergraph via segmentation of the medical imaging exams.

The GRAFIP anatomical model is encoded in a GraphML [24] file structure, which is a comprehensive and easy-to-use standard file format for graphs. Extending the GraphML format with a XSD schema specific to the GRAFIP syntax, it has been possible to describe more complex data structures than in the original GraphML format. More specifically, it was necessary to extend the GraphML schema for two reasons: (1) the high complexity of the brain anatomy entails elaborated node attributes; (2) interaction with the image segmentation module requires specific data structures. GRAFIP encoding in a given hypergraph structure is performed with the open-source Java Universal Network Graph framework library (Section 4.2), implementing extensions of this library in the Eclipse Java development environment. The iterative instantiation of the hypergraph according to MRI segmentation is detailed in the following section.

\subsection{Segmentation of brain MRI data}

Instantiation of the GRAFIP with image-based information requires the segmentation of medical images with dedicated methods. This section summarizes the segmentation framework that has been developed to segment tumors and cerebral structures on brain MRI data.

The segmentation method is based on a recent approach combining parametric or geometric deformable models and spatial constraints $[25,26]$, organized in two phases:

1. Segmentation of the tumors: An initial detection of the tumors is obtained by means of asymmetry analysis and fuzzy clustering mainly based on grey levels, followed by a refinement stage using deformable models [27].

2. Segmentation of the cerebral structures: This process is embedded in a hierarchical construction, where the extraction of a given structure is based on the results of previously segmented structures. The processing of each structure is decomposed into two stages: (i) initialization, which makes extensive use of prior knowledge; (ii) refinement with a 3D deformable model [28], guided by an external force combining a classical data term derived from an edge map of the image data, and a force corresponding to a priori spatial relations with surrounding structures [29]. These spatial relations were modelled in a fuzzy set framework [30]. The proposed combination of spatial relations and deformable models has proved to be very useful to segment cerebral structures with partly occluded edges or very low image contrast, improving segmentation accuracy.

A detailed description of the segmentation procedures is outside the scope of this paper, but can be found in [26,27,29]. This process is pathology-dependent. In particular the spatial relations used for segmenting internal gray nuclei are adapted depending on the type of tumor (the tumor is segmented first and its type is assessed, in particular in terms of spatial extension and induced deformations on the other brain structures). For instance, tumors inducing little deformation will not lead to a modification of the spatial relations used for the segmentation with respect to the ones used in the normal cases. On the contrary, tumors inducing strong deformations will lead to adaptation of the spatial relations, especially metrical ones [31].

This hierarchical approach performs very accurately and robustly for segmentation of normal and pathological brain structures as illustrated in Fig. 4, for a brain tumor, the lateral ventricles and the caudate nuclei. Along the sequential segmentation process, the GRAFIP is refined with specific and precise information describing the segmented structures (e.g. spatial location, shape, size, etc.). More specifically, interactions between the construction of the GRAFIP and the image segmentation process are bidirectional:

- The GRAFIP, representing generic knowledge is used to drive the segmentation, in particular via a priori spatial relations encoded in the hyperedges.

- Conversely, once the segmentation is performed, the GRAFIP is instantiated and modified according to the 
information extracted from the different segmented cerebral structures.

Hence, along the segmentation process, the generic GRAFIP model becomes individualized and patient specific.

The main interest of the GRAFIP is to provide a unified representation framework for both generic knowledge and individual information (specific to the patient under consideration). It allows representing into a single structure, knowledge and information of different natures, including structural information which is not easy to assess without a graph representation. Another feature of the GRAFIP is to facilitate the understanding of the image content, the navigation in the patient's data,

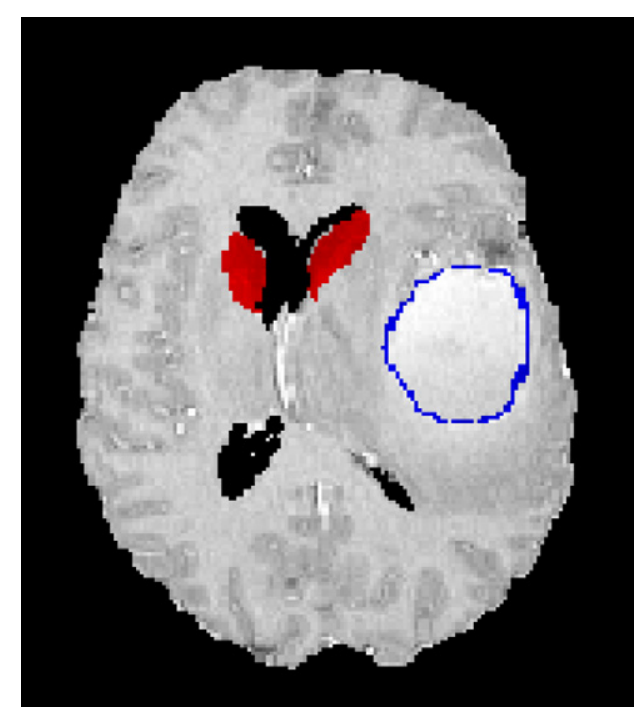

Fig. 4. Brain MRI segmentation of several structures: a tumor (right side closed contour), lateral ventricles, and caudate nuclei (segmented components in the middle). and the visualization of information attached to each image component. Additionally, it can serve as a support for comparison between several images of a patient or of different patients, which is also a complex task that can benefit from the proposed representation.

Appraising the pathology progression requires dedicated processing and encoding tools. In the general framework, the impact of the pathology is evaluated based on MRI segmentation results, and encoded using two procedures, as illustrated in Fig. 5.

1. Adding a "pathology" vertex linked to the surrounding cerebral structures.

2. Updating the vertex and edge attributes of the structures affected by the pathology.

\section{System design}

In order to integrate information extracted from medical images into the EPR, an object oriented generic architecture has been designed. Based on a modular structure, it supports the development of complementary "pathology-dependent" components. A prototype has been implemented using multimedia standards and open-source libraries, to provide interactive visualization of the previously described EPR content. This section presents the system design according to user functional requirements, as well as the main encoded features.

\subsection{Functional user requirements}

Clinical specialists need to exploit computerized medical practice support systems and multimedia data components, in an ergonomic, intuitive, and simple manner, designed to facilitate expertise and multimedia data sharing. Nevertheless, answers to these comprehensive requirements are not

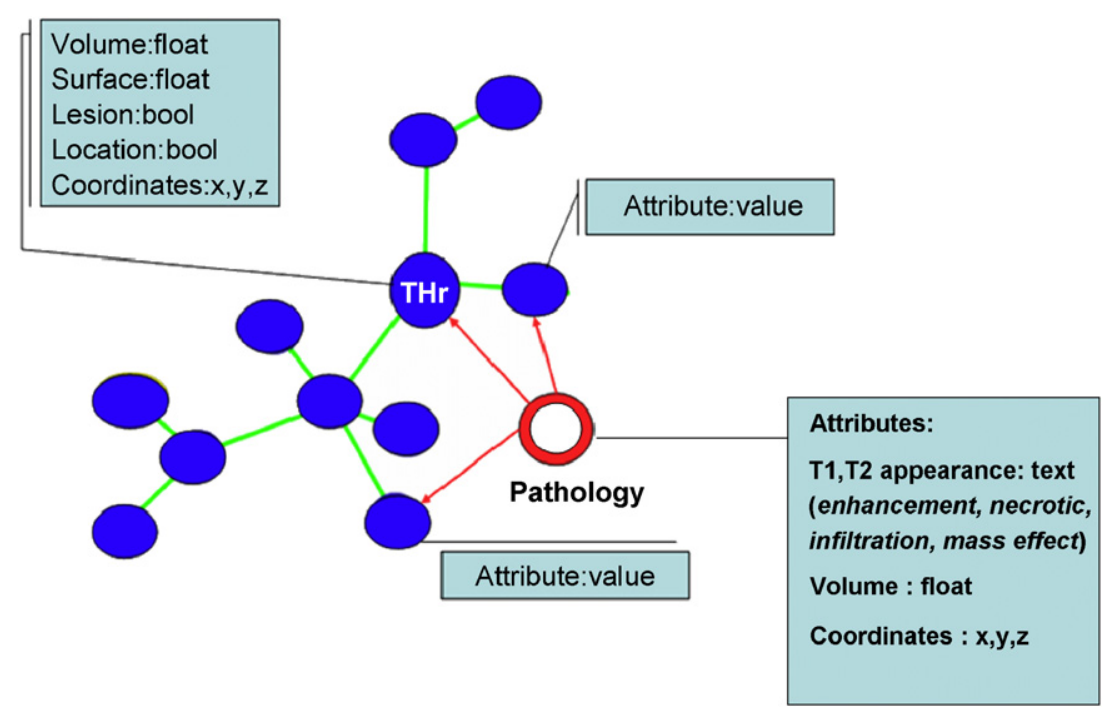

Fig. 5. Pathological graph built from the segmentation of the MRI dataset. Following tumor segmentation, a vertex "tumor" is added and linked to surrounding cerebral structures. Attribute values of the corresponding vertices and edges are then updated. 


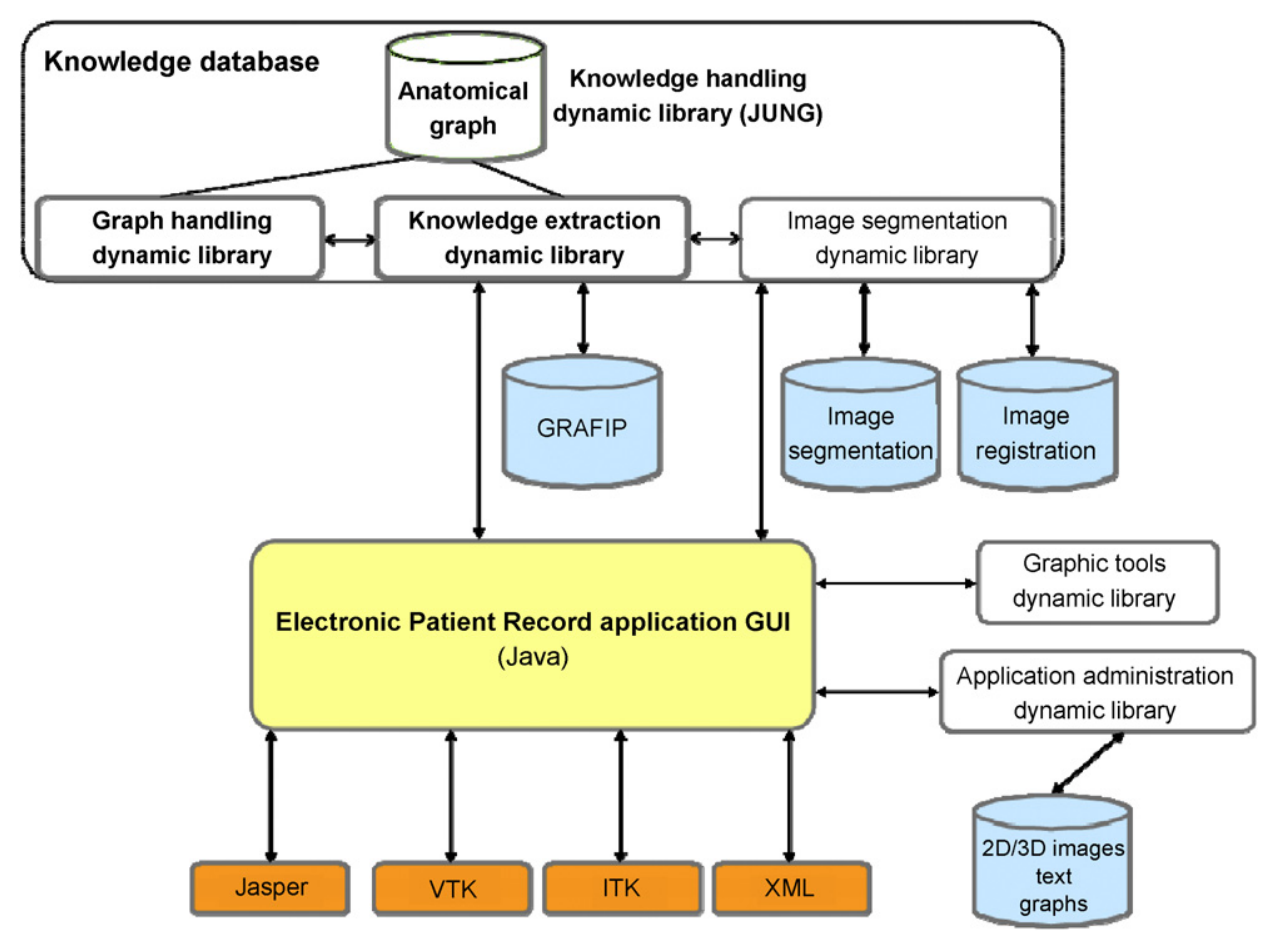

Fig. 6. Main architectural components of the multi-media EPR and their connections.

straightforward, because of numerous constraints: adaptation to the somewhat different specialists' professional culture within different hospitals; isolation of legacy systems; lack of true standards; and economic considerations. In our case the main functional user requirements were primarily identified from previous experience in the conception of EPR-based systems. Given the primary interest on EPR reviewing and MRI navigation tools, specifications focus on user interactivity and integration of image segmentation results into the EPR. The main user requirements can be summarized in a schematic manner as follows:

- There is a single type of user, who is the neurology specialist, even if the system deployment is performed by a system administrator.

- The user interacts exclusively with a GUI, which should be configurable in a flexible manner depending on each EPR characteristics.

- When an EPR is selected, the application displays the available data: administrative information, 2D and/or 3D MRI segmentation results, patient history, and consultation report.

- Spatial relations resulting from the tumor segmentation and surrounding cerebral structures, used to create a patientspecific GRAFIP, can be visualized over the original or segmented images, as edge graph attributes.

- The GRAFIP is integrated into the multimedia EPR after validation from the specialist.

These functionalities led to the specification of the system architecture.

\subsection{System implementation}

In order to cope with all the characteristics of the multimedia EPR, an open source technology framework was identified as the most appropriate. Two reasons motivate this decision. On the one hand, most of the commercially available EPR systems are proprietary and as a consequence, incompatible with other types of complementary platforms. On the other hand, open source technologies, depending on license conditions, can reduce development costs, increasing at the same time the flexibility and the potential extension of the implemented applications. Various open-source libraries were combined in the EPR application development: the JPEG-2000 Jasper codec library (www.ece.uvic.ca/ mdadams/jasper), the Java XML application programming interface, the visualization toolkit VTK (www.vtk.org), the Insight segmentation and registration toolkit (www.itk.org), and the Java Universal Network Graph Framework JUNG (jung.sourceforge.net) that was extended to produce the GRAFIP. Integration development was carried out with the Java Eclipse environment (www.eclipse.org).

Fig. 6 illustrates the different architectural components and associated libraries, along with their interconnections. According to the defined user requirements, a flexible GUI facilitates user interactions, managing the different EPR elements display and storage. Interactive visualization and navigation through displayed patient data is enabled in an intuitive and ergonomic environment.

To illustrate the enhanced capabilities of the adapted 3D navigation GUI, allowing for visualization and quantification of the anatomy and pathological findings, we now present a 


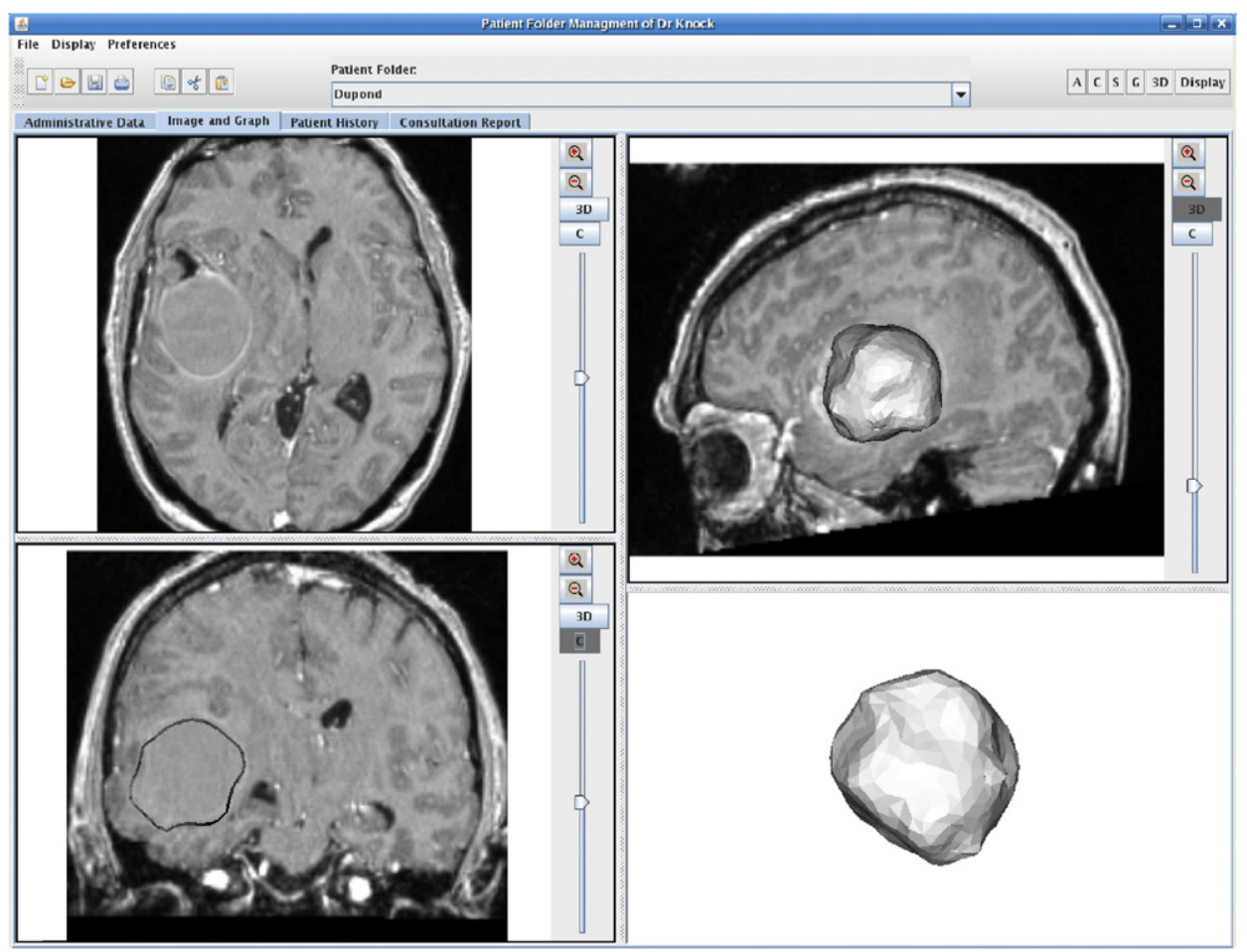

Fig. 7. Image segmentation display combined with MRI data. From left to right and top to bottom: axial view of the imaging data; sagittal view with superposition of the 3D segmented tumor; coronal view with contour of the segmented tumor; and a 3D visualization of the tumor.

neurological examination scenario of brain tumor diagnosis based on: (1) the interpretation of MRI datasets and (2) the review of the GRAFIP information content.

\subsubsection{EPR loading}

Following user identification with the corresponding login and password, a profile adapted interface is displayed. It provides access to a set of EPRs, by means of a scroll-down menu. EPRs are identified by the patients' name, and they contain all the related EPR files stored in a single directory. After a patient has been selected the application reads all the EPR content: administrative information, 2D/3D images, 2D/3D segmentation results, GRAFIP, patient history, and consultation reports. DICOM image volumes, jpeg or raw image sequences are read by the application; any other format can be read as long as the corresponding plugging is added. At the present stage of the application development, the medical expert does not activate contour segmentation, but previously generated segmentation results are available when a patient record is accessed. At this point, we have separated the representation task and the segmentation task. One of the reasons is that segmentation is still a difficult task, which did not find a definite and automated solution in the literature until now. Moreover imaging techniques as well as image processing methods evolve rapidly, while the anatomy is a quite established knowledge. One of the advantages of keeping representation and segmentation separated is that any segmentation input can serve as an input to instantiate the GRAFIP, thus achieving a better modularity of the whole system. However, it should be mentioned that the knowledge encoded in the GRAFIP is intensively used in the segmentation process, and that segmentation results are directly incorporated into the individualized GRAFIP. Thus strong links already exist between both elements.

\subsubsection{EPR data}

In order to provide simple interactions with the displayed EPR data, different data categories have been defined and displayed in four separated tabbed panels. These categories are: administrative data, images and graph, patient history, and consultation reports.

The data size of the whole EPR dataset is frequently larger than the application's memory capabilities. To handle this limitation, a tool bar allows the selection of a specific dataset to visualize at a particular time. Therefore, a set of icons (letters displayed on the top right part of the GUI) is provided to interactively define multiple datasets display when required. Likewise, the user can configure the navigation tools, according to the desired degree of details, integrating clinical data, 2D and 3D images, 2D or 3D segmented tumors and surrounding anatomical structures, along with the patient-specific GRAFIP.

\subsubsection{Image and segmentation results display}

Segmentation results are stored in separate volumes of labeled data, with a predefined filename corresponding to known cerebral structures or lesions (e.g. tumor, right_ventricle, right_caudale_nucleus). A list of segmented structures available 
in the EPR is reviewed by the expert user who selects the ones that should be displayed.

The diagnosis can be improved by presenting all available data to the physician for a complete review. Combined visualization of T1 and T2 weighted MRI data, with and without contrast, may enable a more precise definition of the tumor boundaries and the associated edema (from T2-w), as well as the tumor's active part and necrotic part (from T1-w exams with contrast).

An example of an interactive EPR visualization combining MRI data and tumor segmentation results is displayed in Fig. 7.

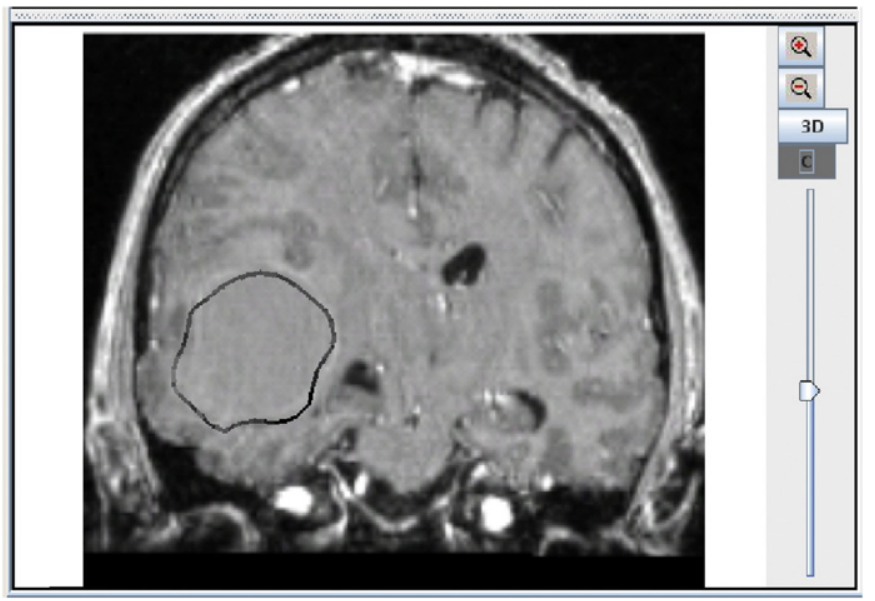

Fig. 8. MRI coronal slice and contour of the segmented tumor. Interaction tools represented by icons are displayed on the right side of the panel (in descending order): zoom-in, zoom-out, 3D visualization of the segmented object, and segmented contours.
On the other hand, individual data display windows can be adapted to different visualization requirements as illustrated in Fig. 8, with complementary user interaction tools such as zooms and toggle of 2D or 3D segmentation display. More specifically, it is possible to navigate through the image volume with a slider, add either the 3D segmented lesion or its intersection with the current slice, as well as carry out local visualization using a "zoom in", or step backwards using a "zoom out". Furthermore, when the 3D object is separately displayed, the user can examine the surface from any point of view. The rest of the EPR content remains available during image visualization and navigation.

\subsubsection{Image and GRAFIP display and navigation}

Tumor positioning, along with the identification of the related anatomical and pathological structures surrounding it, give to the neurologist a complete topographical description of the tumor. The associated GRAFIP provides this information in a structured manner: lesion size, presence of edema or cyst, spatial relations resulting from the tumor segmentation and surrounding cerebral structures, and anatomical and functional localization of the pathology according to atlases (e.g. Montreal Neurological Institute atlas and Broadman reference). The described visualization and navigation tool combines MRI image volumes, tumor segmentation results, GRAFIP display, consultation reports, and patient data for clinical reviewing purposes.

Possible GUI configurations include 1, 2, 3, or 4 display panels (Fig. 7) to choose from, corresponding to axial (A), coronal (C), or sagittal (S) views, combined with graph visualization (G) and 3D lesion display (3D). In Fig. 9 an example of an axial

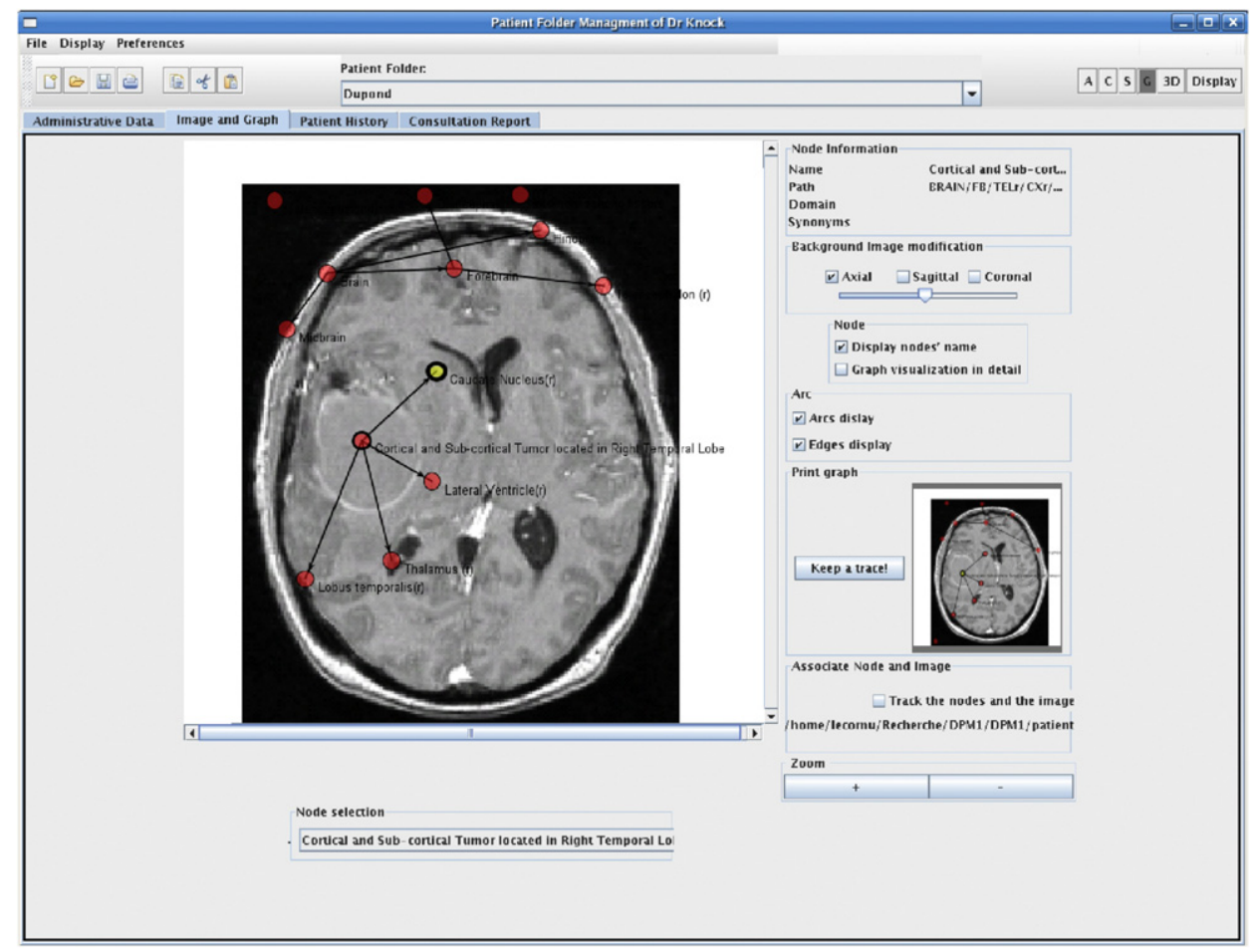

Fig. 9. GRAFIP display combined with MRI data. By default the axial view is displayed, even though the sagittal or coronal views can be selected afterwards. 


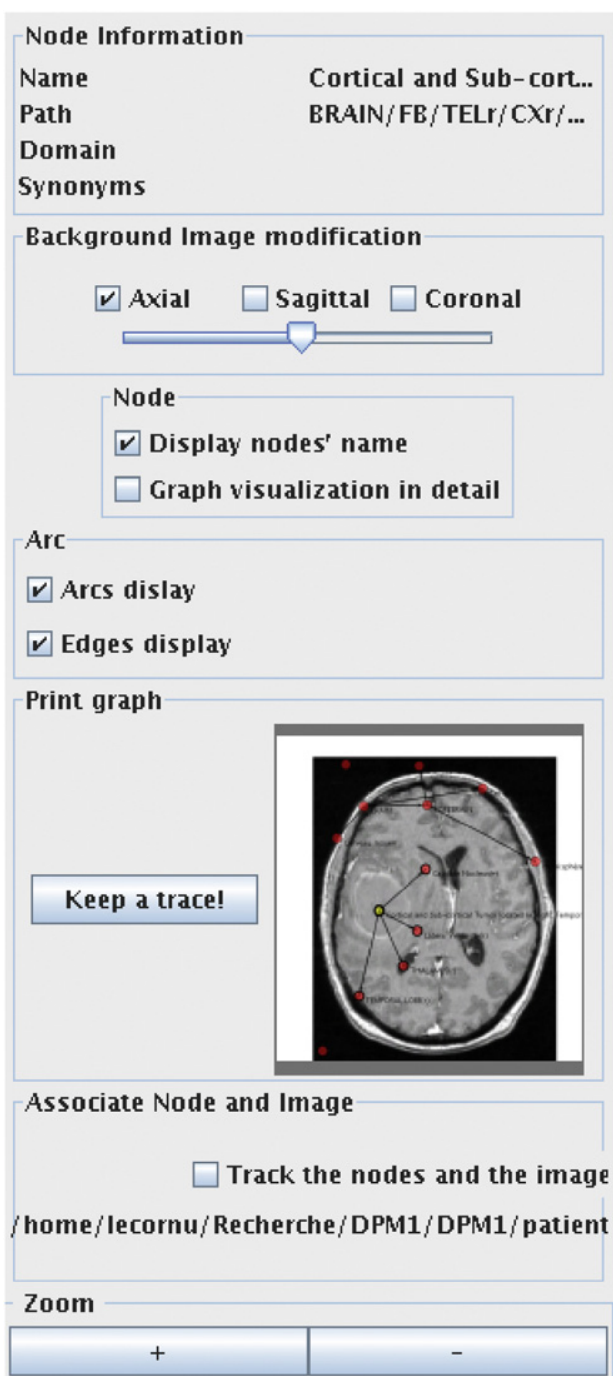

Fig. 10. Parameters for the GRAFIP interactive display. Besides the node information display, it is possible to change the visualized image volume projection, add information to the nodes presentation (name and details), decide to draw the lines connecting the nodes (Arcs display), and/or their direction (Edges display), keep a graphic trace of the obtained GRAFIP and image superposition, eventually recording the physical positions of the nodes on the image (track the nodes and the image), or regulate the visualized image detail with a zoom.

MRI slice is provided along with the corresponding GRAFIP, built from the segmented cerebral structures.

Parameters for the graph display are selected on the right side of the GUI control window (Fig. 10). Those parameters include:

- The interactively selected node, list of information fields (name, hierarchical path, domain, and synonyms).

- The possibility to toggle the MRI choosing one of three standard orthogonal views.

- The option to enhance the display of node's names or instantiated graph details, as well as the arcs between nodes.

- The choice to save a snapshot of the generated GRAFIP displayed with the image, independently, or by sections at different resolution levels.
The clinician has the possibility to modify or complete the GRAFIP. Afterwards, if the specialist validates it, the GRAFIP is integrated to the corresponding multimedia EPR.

\section{Discussion and conclusions}

The proposed EPR application prototype offers enhanced clinical information exploitation capabilities, which are useful for therapeutic patient follow-up, and could eventually lead to original changes in the way medical information is exploited. Based on multiple image formats, combined with text and graph data formatted in XML it provides an ergonomic GUI design capable of displaying all associated data and information in a simple and practical manner, along with segmented anatomical structures. A preliminary evaluation of the EPR application with three neurologists provided a positive feedback concerning the application capabilities and precise indications for its evolution. Compared to available applications, the proposed prototype has the advantage of combining in a practical way images and clinical data, instead of handling them separately, using at least two different visualization applications. Regarding the integration of the GRAFIP with the image data, graph nodes display offers the possibility of identifying specific anatomical zones to protect during a therapy procedure (for instance, radiation or surgery), or an invasive intervention, along with the display of related functional information. Being able to visualize a significant amount of data and information simultaneously, combined with flexible user interactions, simplifies the patient data and medical imaging examination review and interpretation tasks. Additionally, the configurable GUI allows examining patient multimedia data according to the specialist's preferences.

The system will be further developed to be used for indexing, and longitudinal clinical case study, using databases of MRI exams previously reviewed by medical experts. In this context, the interface will provide an ergonomic and rapid reviewing tool of complete EPR data for the neurologist, with the possibility of querying in a common database for patients with similar lesions. Some improvements in the interaction capabilities provided by the interface are still required, including the authorized creation of new patient records, the modification of segmentation results by means of medical expert adjustments, the expansion of the EPR content, as well as security tools for shared use of the EPR system by different experts. Applicability to other areas depends mainly on the development of anatomical and functional information graphs, to be used for generating patient-specific GRAFIP instances associated to the segmented normal and pathologic structures. On the other hand, modifications would be required at the data model and EPR navigation levels, according to users' functional requirements. Another promising extension concerns other types of pathologies such as strokes: the GRAFIP unified representation allows associating a specific deficit to a particular cerebral localization of the lesion. This anatomo-functional question and its associated anatomo-functional representation could lead to a natural and important evolution of the GRAFIP. 


\section{Summary}

Several medical imaging modalities are playing an increasingly important role in diagnosis, leading to significant modifications in clinical practice and multimedia data utilization. However, up to now, electronic patient record (EPR) evolution has followed a slower track compared to healthcare costs analysis and management systems. Even if the integration of medical images in the EPR is being handled at certain levels by some medical information systems, image segmentation and knowledge extracted from medical images are not integrated. This paper presents a contribution to this large problematic, focusing on the integration into the EPR of: (1) information extracted from medical images (e.g. MRI segmentation) and (2) prior anatomical knowledge by means of a graph of representation of anatomical and functional information for individual patients including pathologies (GRAFIP). Brain tumor therapy planning and follow-up was chosen as context, to design a multimedia EPR system integrating a reviewing application prototype. The proposed information integration system offers enhanced capabilities for reviewing clinical information extracted from medical images, toward an integrated therapeutic follow-up tool. Among rich interaction possibilities, an intuitive and flexible graphic user interface, visualization and navigation through $2 \mathrm{D}$ and $3 \mathrm{D}$ brain images, tumor segmentation, association of the obtained results to anatomical knowledge, and an enhanced visualization of the specific knowledge in the form of a superposed graph are simultaneously available. Open source technology was chosen for prototype implementation to facilitate interoperability with other complementary platforms, and to increase the flexibility of the developed application, while reducing development costs. The prototype offers a practical tool for advantageous combinations of images and clinical data. Being able to visualize a significant amount of data and information simultaneously, combined with flexible user interactions, it simplifies tremendously the task of reviewing and interpreting medical imaging examinations, as the clinical expert can handle patient data in an ergonomic and efficient manner. Several expert neurologists evaluated the proposed architecture and functionalities of the multimedia EPR system, providing a positive feedback and indications for its evolution. Further improvements of the system will be carried out for indexation, and longitudinal clinical cases studies, using databases of MRI exams previously reviewed by medical experts.

\section{Conflict of interest statement}

None declared.

\section{Acknowledgements}

This work was partially funded by an Institut TELECOM grant. The authors wish to thank Dr. L. Capelle from Hôpital Pitié-Salpêtriere for providing the database elements, Dr. B. Dubois, Dr. H. Duffau, Dr. E. Mandonnet, and Dr. D. Hasboun from Hôpital Pitié-Salpêtrière, and Dr. B. Devaux,
Mr. R. Clodic, and Mr. M. Harislur from the Centre Hospitalier de Sainte Anne in Paris, for their participation in the project.

\section{References}

[1] M. Wang, C. Lau, F.A. Matsen, Y. Kim, Personal health information management system and its application in referral management, IEEE Trans. Inf. Technol. Biomed. 8 (3) (2004) 287-297.

[2] M.C. Beuscart-Zéphir, F. Anceaux, V. Crinquette, J.M. Renard, Integrating users' activity modelling in the design and assessment of hospital electronic patient records: the example of anesthesia, Int. J. Med. Inf. 64 (2001) 157-171.

[3] P.J.B. Brown, V. Warmington, Data quality probes-exploiting and improving the quality of electronic patient record data and patient care, Int. J. Med. Inf. 68 (3) (2005) 91-98.

[4] M.Y.Y. Law, A model of DICOM-based electronic patient record in radiation therapy, Computerized Med. Imaging Graphics 29 (2-3) (2005) 125-136.

[5] F. Malamateniou, G. Vassilacopoulos, Developing a virtual patient record using XML and web-based workflow techniques, Int. J. Med. Inf. 70 (3) (2003) 131-139.

[6] D.P. Lorence, R. Churchill, Clinical knowledge management using computerized patient record systems: Is the current infrastructure adequate?, IEEE Trans. Inf. Technol. Biomed. 9 (2) (2005) 283-288.

[7] M.Y.Y. Law, H.K. Huang, Concept of a PACS and imaging informaticsbased server for radiation therapy, Computerized Med. Imaging Graphics 27 (1) (2003) 1-9.

[8] O. Ratib, M. Swiernik, J.M. McCoy, From PACS to integrated EMR, Computerized Med. Imaging Graphics 27 (3) (2003) 207-215.

[9] N.-T. Cheung, A. Lam, W. Chan, J.H.B. Kong, Integrating images into the electronic patient record of the hospital authority of Hong Kong, Computerized Med. Imaging Graphics 29 (2-3) (2005) 137-142.

[10] J. Zhang, J. Sun, Y. Yang, X. Chen, L. Meng, P. Lian, Webbased electronic patient records for collaborative medical applications, Computerized Med. Imaging Graphics 29 (2-3) (2005) 115-124.

[11] T. Amouh, M. Gemo, B. Macq, J. Vanderdonckt, A.W. El Gariani, M.S. Reynaert, L. Stamatakis, F. Thys, Versatile clinical information system design for emergency departments, IEEE Trans. Inf. Technol. Biomed. 9 (2) (2005) 174-183.

[12] S.S. Furuie, M.S. Rebelo, R.A. Moreno, M. Santos, N. Bertozzo, G.H Motta, F.A. Pires, M.A. Gutierrez, Managing medical images and clinical information: InCor's experience, IEEE Trans. Inf. Technol. Biomed. 11 (1) (2007) 17-24.

[13] S. Cha, Update on brain tumor imaging: from anatomy to physiology, Am. J. Neuroradiol. 27 (3) (2006) 475-487.

[14] T. Beale, The health record-why is it so hard?, in: R. Haux, C. Kulikowski (Eds.), IMIA Yearbook of Medical Informatics 2005: Ubiquitous Health Care Systems, Stuttgart, Schattauer, 2005, pp. 301-304.

[15] I.M. Mullins, M.S. Siadaty, J. Lyman, K. Scully, C.T. Garrett, W.G. Miller, R. Muller, B. Robson, C. Apte, S. Weiss, I. Rigoutsos, D. Platt, S. Cohen, W.A. Knaus, Data mining and clinical data repositories: insights from a 667,000 patient data set, Comput. Biol. Med. 36 (12) (2006) $1351-1377$.

[16] D.F. Leotta, Y. Kim, Requirements for picture archiving and communications, IEEE Eng. Med. Biol. Mag. 12 (1) (1993) 62-69.

[17] P.C. Cosman, R.M. Gray, R.A. Olshen, Evaluating quality of compressed medical images: SNR, subjective rating, and diagnostic accuracy, Proc. IEEE 82 (6) (1994) 919-932.

[18] S.-G. Miaou, S.-T. Chen, Automatic quality control for wavelet-based compression of volumetric medical images using distortion-constrained adaptive vector quantization, IEEE Trans. Med. Imaging 23 (11) (2004) 1417-1429.

[19] C. Christopoulos, A. Skodras, T. Ebrahimi, The JPEG 2000 still image coding system: an overview, IEEE Trans. Consum. Electron. 46 (4) (2000) 1103-1127.

[20] L. Montesinos, J. Puentes, Specialized telepathology electronic patient record based on JPEG 2000, in: Proceedings of the International IEEE 
EMBS Conference: Special Topic Conference on Information Technology Applications in Biomedicine, 2003, pp. 110-113.

[21] B. Batrancourt, J. Atif, O. Nempont, E. Angelini, I. Bloch, Integrating information from pathological brain MRI into an anatomo-functional model, in: Proceedings of the 24th IASTED International Conference on Biomedical Engineering, 2006, pp. 236-241.

[22] D.M. Bowden, R.F. Martin, Neuronames brain hierarchy, NeuroImage 2 (1) (1995) 63-83.

[23] K. Brodmann, Vergleichende Lokalisationlehre der Grosshirnrinde in ihren Prinzipien dargestellt auf Grund des Zellenbaues, Leipzig, Germany, Barth, 1909.

[24] U. Brandes, M. Eiglsperger, I. Herman, M. Himsolt, M.S. Marshall, Graphml progress report: structural layer proposal, in: Proceedings of the 9th International Symposium on Graph Drawing, Lecture Notes in Computer Science, vol. 2265, 2002, pp. 501-512.

[25] O. Colliot, O. Camara, I. Bloch, Integration of fuzzy structural information in deformable models, in: Proceedings of the Information Processing and Management of Uncertainty (IPMU), 2004 pp. $1533-1540$.

[26] J. Atif, O. Nempont, O. Colliot, E. Angelini, I. Bloch, Level set deformable models constrained by fuzzy spatial relations, in: Proceedings of the Information Processing and Management of Uncertainty (IPMU), 2006, pp. 1534-1541.

[27] H. Kothanlou, J. Atif, O. Colliot, I. Bloch, 3D brain tumor segmentation using fuzzy classification and deformable models, in: Proceedings of the International Workshop on Fuzzy Logic and Applications, 2005, pp. 312-318.

[28] C. Xu, D.L. Pham, J.L. Prince, Image segmentation using deformable models, in: Handbook of Medical Imaging, vol. 2, Medical Image Processing and Analysis, SPIE Press, 2000, pp. 131-169.

[29] O. Colliot, O. Camara, I. Bloch, Integration of fuzzy spatial relations in deformable models: application to brain MRI segmentation, Pattern Recognition 39 (8) (2006) 1401-1414.

[30] I. Bloch, Fuzzy spatial relationships for image processing and interpretation: a review, Image Vision Comput. 23 (2) (2005) 89-110.

[31] H. Khotanlou, J. Atif, E. Angelini, H. Duffau, I. Bloch, Adaptive segmentation of internal brain structures in pathological MR images depending on tumor types, in: Proceedings of the 4th International Symposium on Biomedical Imaging: From Nano to Macro, ISBI, 2007, pp. 588-591.

John Puentes holds an electronics engineering degree, and an M.Sc. of image processing and artificial intelligence. He also holds a Ph.D. in signal processing and telecommunications from the Rennes I University, France gained in 1996. He worked as an engineer, consultant, and project manager for biomedical and telecommunications companies, before moving to the Image and Information Processing Department at TELECOM Bretagne, where he is an assistant professor and an associate researcher of the French Institute of Health and Medical Research (INSERM). His primary research interests are medical decision support systems, image indexing, and the application of multimedia emerging technologies for medical information processing.

Bénédicte Batrancourt was born in Molain, France in 1961. She received a Ph.D. from the Claude Bernard University, Lyon 1, France, in 2003 Since 2006, she has been with the Pitié Salpêtrière Hospital in Paris, where she is a Postdoc in INSERM U610 unit (functional neuro-anatomy of the behaviour and its disorders). Her research interests focus on human brain mapping, structure-function relations, and the framework to the study of the anatomical networks in the human brain. Her actual project is the definition of a structured database of focal brain lesions, and of the methods to obtain a homogeneous database means to get homogeneous data, for in the final to establish explore the relationships between patterns of neuropsychological impairment and specific brain lesions groups of specific patients in a particular anatomical-functional field.

Jamal Atif was born in Rabat, Morocco, in 1978. He received a master degree from the University of Paris-XI, in 2000 and a Ph.D., from the same university, in 2004. Since 2005, he has been with TELECOM Paris Tech as a postdoctoral fellow. In 2006, he joined the Université des Antilles et de la Guyane and IRD-Cayenne, Unité ESPACE as an assistant professor. His research interests focus on image processing and understanding and graphbased knowledge representation and reasoning.

Elsa Angelini received an engineering degree from Ecole Centrale de Nantes France, in 1996 and a Ph.D. degree in Biomedical Engineering from Columbia University, USA, in 2003. Since 2004, she is a professor in the Department of Signal and Image Processing at, TELECOM Paris Tech, France. Her research interests focus on medical image processing and anatomical modeling.

Laurent Lecornu was born in Rennes in 1967. He holds an M.Sc. in signal processing and telecommunications from the Rennes I University, France, gained in 1990. He also holds a Ph.D. in signal processing and telecommunications from the same university, gained in 1995. Lecornu is an assistant professor in the Image and Information Processing Department at TELECOM Bretagne, Since 2002, and a member of the LaTIM, INSERM U650. His primary research interests are image processing, image indexing, and medical databases data mining.

Abdelhamid Zemirline received an M.Sc. degree in Computer Science from the University of Aix Marseille III, France, in 2003. He is currently a Ph.D. student at the Department of Image and Processing Information, TELECOM Bretagne, France. His research interests include data fusion, machine learning, data mining, artificial intelligence, and medical diagnostic support systems.

Isabelle Bloch is a professor at TELECOM Paris Tech (Signal and Image Processing Department). Her research interests include 3D image and object processing, 3D and fuzzy mathematical morphology, decision theory, information fusion, fuzzy set theory, belief function theory, structural and graph-based pattern recognition, spatial reasoning, and medical imaging.

Gouenou Coatrieux holds an engineer degree in electronics and industrial informatics from the École Nationale Supérieure des Sciences Appliquées et de Technologie, Lannion France, and an M.Sc. degree in signal, telecommunications, image, and radar from Rennes I University, France, both gained in 1999. He also holds a Ph.D. in signal processing and telecommunications from the University of Rennes I, France, obtained in 2002. Coatrieux is currently an assistant professor in the Image and Information Processing Department, at TELECOM Bretagne, and member of the LaTIM, INSERM U650 laboratory. His primary research interests concern medical information systems security and image watermarking.

Christian Roux graduated from the Ecole Normale Supérieure de Cachan, France, in 1978 and received the Ph.D. degree in image processing from the Institut National Polytechnique de Grenoble, France, in 1980. He is a professor in the Image and Information Processing Department of TELECOM Bretagne. He is the director of LaTIM. His main research interests are medical imaging and information processing. 